\title{
Toward Full Spatio-Temporal Control on the Nanoscale
}

\author{
Maxim Durach, ${ }^{1}$ Anastasia Rusina, ${ }^{1}$ Keith Nelson, ${ }^{2}$ and Mark I. Stockman ${ }^{1}$ \\ 1 Department of Physics and Astronomy, Georgia State University, Atlanta, Georgia 30303, USA \\ 2 Department of Chemistry, MIT, Cambridge, MA 02139, USA
}

(Dated: October 31, 2018)

\begin{abstract}
We introduce an approach to implement full coherent control on nanometer length scales. It is based on spatio-temporal modulation of the surface plasmon polariton (SPP) fields at the thick edge of a nanowedge. The SPP wavepackets propagating toward the sharp edge of this nanowedge are compressed and adiabatically concentrated at a nanofocus, forming an ultrashort pulse of local fields. The one-dimensional spatial profile and temporal waveform of this pulse are completely coherently controlled.
\end{abstract}

PACS numbers: 78.67.-n, 71.45.Gm, 42.65.Re, 73.20.Mf

Two novel areas of optics have recently attracted a great deal of attention: nanooptics and ultrafast optics. One of the most rapidly developing directions in ultrafast optics is quantum control, in which coherent superpositions of quantum states are created by excitation radiation to control the quantum dynamics and outcomes $1,2,3,4$. Of special interest are coherently controlled ultrafast phenomena on the nanoscale where the phase of the excitation waveform along with its polarization provides a functional degree of freedom to control nanoscale distribution of energy ${ }^{5,6,7,8,9,10}$. Spatiotemporal pulse shaping permits one to generate dynamically predefined waveforms modulated both in frequency and in space to focus ultrafast pulses in the required microscopic spatial and femtosecond temporal domains ${ }^{11,12}$.

In this Letter, we propose and theoretically develop a method of full coherent control on the nanoscale where a spatiotemporally modulated pulse is launched in a graded nanostructured system. Its propagation and adiabatic concentration provide a possibility to focus the optical energy in nanoscale spatial and femtosecond temporal regions. The idea of adiabatic concentration ${ }^{13,14}$ (see also Ref. 15) is based on adiabatic following by the propagating surface plasmon-polariton (SPP) wave of a plasmonic waveguide, where the phase and group velocities decrease toward a limit at which the propagating SPP wave is adiabatically transformed into a standing surface plasmon (SP) mode. This effect has been further developed theoretically $\underline{\underline{16}, 17}$ and observed experimentally, $\underline{\underline{18}}$

To illustrate the idea of this full coherent control, consider first the adiabatic concentration of a plane SPP wave propagating along a nanowedge of silver ${ }^{19}$, as shown in Fig. 1(a); the theory is based on the Wentzel-KramersBrillouin (WKB) or quasiclassical approximation, also called the eikonal approximation in optics ${ }^{20}$ as suggested in Refs. 13 14. The propagation velocity of the SPP along such a nanowedge is asymptotically proportional to its thickness. Thus when a SPP approaches the sharp edge, it slows down and asymptotically (in the ideal limit of zero thickness at the apex) stops while the local fields are increased and nano-concentrated.

Now consider a family of SPP rays (WKB trajectories) propagating from the thick side of a nanowedge,

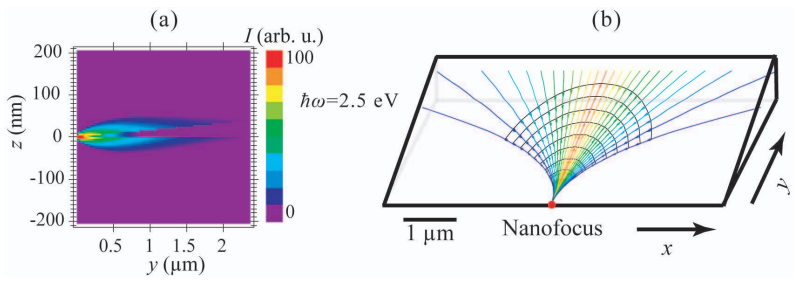

FIG. 1: (a) Illustration of adiabatic concentration of energy on the wedge. The distribution of local field intensity $I$ in the normal plane of propagation of SPPs (the $y z$ plane). The intensity in relative units is color coded with the color scale bar shown to the right. (b) Trajectories of SPP rays propagating from the thick to sharp edge of the wedge. The initial coordinate is coded with color. The black curves indicate lines of equal phase (SPP wave fronts).

as shown in Fig. 1(b). One possibility to launch such SPPs is to have nanoscale inhomogeneities (nanoparticles or nanoholes) at the thick edge of the wedge. Each of the optical pulses from the spatiotemporal shaper is focused on the corresponding nanoparticle, scattering from it and generating an SPP wave. This scattering is necessary to impart large transverse momenta on SPPs, which is required for their efficient transverse focusing. These SPP waves propagate toward the sharp edge, adiabatically slow down which increases the field amplitudes, and constructively interfere as they converge at the nanofocus.

The phases of the SPPs rays (i.e., the corresponding wave fronts) are defined by the spatiotemporal modulator in such a way that the rays converge to a reconfigurably chosen point at the sharp edge of the nanowedge where they acquire equal phases. When the SPPs propagate along the rays, adiabatic concentration takes place because the SPP wavelength tends to zero proportionally to the thickness of the wedge. This allows one to focus optical energy at a predefined nanofocus at the sharp edge. The temporal structure of the generated SPPs can be chosen in such a way that at the nanofocus the local fields form an ultrashort pulse. Using spatiotemporal modulation of the excitation field at the thick edge, one can arbitrarily move the nanofocus along the sharp edge. 
A superposition of such fields can render arbitrary spatiotemporal modulation on the sharp edge, enabling one to exert full control over nanoscale fields in space and time.

Turning to the theory, consider a nanofilm of metal in an $x y$ plane whose thickness $d$ in the $z$ direction is adiabatically changing with the coordinate-vector $\boldsymbol{\rho}=$ $(x, y)$ in the plane of the nanofilm. Let $\varepsilon_{m}=\varepsilon_{m}(\omega)$ be the dielectric permittivity of this metal nanofilm, and $\varepsilon_{d}$ be the permittivity of the embedding dielectric. Because of the symmetry of the system, there are odd and even (in the normal electric field) SPPs. It is the odd SPP that is a slow-propagating, controllable mode. The dispersion relation for this mode defining its effective index $n(\boldsymbol{\rho})$ is

$$
\tanh \left(\frac{1}{2} k_{0} d(\boldsymbol{\rho}) \sqrt{n(\boldsymbol{\rho})^{2}-\varepsilon_{m}}\right)=-\frac{\varepsilon_{d} \sqrt{n(\boldsymbol{\rho})^{2}-\varepsilon_{m}}}{\varepsilon_{m} \sqrt{n(\boldsymbol{\rho})^{2}-\varepsilon_{d}}},
$$

where $k_{0}=\omega / c$ is the radiation wave vector in vacuum.

Let $\boldsymbol{\tau}$ be a unit tangential vector to the SPP trajectory (ray). It obeys a conventional equation of ray optics ${ }^{20}$

$$
n \frac{d \boldsymbol{\tau}}{d l}=\nabla n-\boldsymbol{\tau}(\boldsymbol{\tau} \nabla n),
$$

where $l$ is the length along the ray and $\nabla=\partial / \partial \rho$.

Now let us consider a nanofilm shaped as a nanowedge as in Fig. 1(b). In such a case, $n=n(y)$, and these trajectory equations simplify as

$$
n \frac{d \tau_{y}}{d l}=\tau_{x}^{2} \frac{\partial n}{\partial y}, \quad n \frac{d \tau_{x}}{d l}=-\tau_{x} \tau_{y} \frac{\partial n}{\partial y} .
$$

From these, it follows that $n_{x} \equiv \tau_{x} n=$ const. The SPP wave vector, related to its momentum, is $\mathbf{k}(\boldsymbol{\rho})=$ $k_{0} n(\boldsymbol{\rho}) \boldsymbol{\tau}$; this is the conservation of $k_{x}$ (the transverse momentum). This allows one to obtain a closed solution for the ray. The tangent equation for the ray is $d x / d y=\tau_{x} / \tau_{y}$, where $\tau_{y}=\sqrt{1-n_{x}^{2} / n^{2}}$. From this, we get an explicit SPP trajectory (ray) equation as

$$
x-x_{0}=\int_{y_{0}}^{y}\left(\frac{n\left(y^{\prime}\right)^{2}}{n_{x}^{2}}-1\right)^{-1 / 2} d y^{\prime},
$$

where $\boldsymbol{\rho}_{0}=\left(x_{0}, y_{0}\right)$ is the focal point where rays with any $n_{x}$ converge. To find the trajectories, as $n(y)$ we use the real part of effective index (1), as WKB suggests.

When the local thickness of the wedge is subwavelength $\left(k_{0} d \ll 1\right)$, the form of these trajectories can be found analytically. Under these conditions, dispersion relation (1) has an asymptotic solution

$$
n=\frac{n_{a}}{k_{0} d}, \quad n_{a}=\ln \frac{\varepsilon_{m}-\varepsilon_{d}}{\varepsilon_{m}+\varepsilon_{d}} .
$$

Substituting this into Eq. (4), we obtain explicit equations of trajectories,

$$
\left(x-x_{0}-\sqrt{\frac{\bar{n}_{a}^{2}}{n_{x}^{2}}-y_{0}^{2}}\right)^{2}+y^{2}=\frac{\bar{n}_{a}^{2}}{n_{x}^{2}},
$$

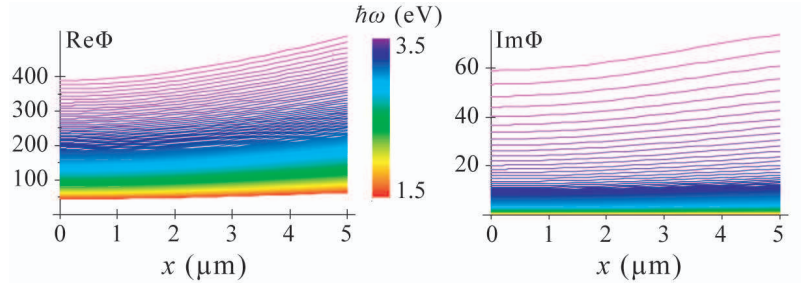

FIG. 2: (a) Phase (real part of eikonal $\Phi$ ) acquired by a SPP ray propagating between a point with coordinate $x$ on the thick edge and the nanofocus, displayed as a function of $x$. The rays differ by frequencies that are color coded by the vertical bar. (b) The same as (a) but for extinction of the ray $(\operatorname{Im} \Phi)$.

where $\bar{n}_{a}=n_{a} /\left(k_{0} \tan \theta\right)$, and $\tan \theta$ is the slope of the wedge. Thus, each SPP ray is a segment of a circle whose center is at a point given by $x=x_{0}+\sqrt{\left(\bar{n}_{a} / n_{x}\right)^{2}-y_{0}^{2}}$ and $y=0$. This analytical result is in agreement with Fig. 1 (b). If the nanofocus is at the sharp edge, i.e., $y_{0}=0$, then these circles do not intersect but touch and are tangent to each other at the nanofocus point.

As an example we consider a silver $\frac{19}{19}$ nanowedge illustrated in Fig. 1(b) whose maximum thickness is $d_{m}=30$ $\mathrm{nm}$ and whose length (in the $y$ direction) is $L=5 \mu \mathrm{m}$. Trajectories calculated from Eq. (4) for $\hbar \omega=2.5 \mathrm{eV}$ are shown by lines (color used only to guide eye); the nanofocus is indicated by a bold red dot. The different trajectories correspond to different values of $n_{x}$ in the range $0 \leq n_{x} \leq n(L)$. In contrast to focusing by a conventional lens, the SPP rays are progressively bent toward the wedge slope direction.

The eikonal is found as an integral along the ray

$$
\Phi(\boldsymbol{\rho})=\int_{\boldsymbol{\rho}_{0}}^{\boldsymbol{\rho}} \mathbf{n}(\boldsymbol{\rho}) d \boldsymbol{\rho} .
$$

Consider rays emitted from the nanofocus [Fig. 1 (b)]. Computed from this equation for frequencies in the visible range, the phases of the SPPs at the thick edge of the wedge (for $y=L$ ) are shown in Fig. 2 (a) as functions of the coordinate $x$ along the thick edge. The colors of the rays correspond to the visual perception of the ray frequencies. The gained phase dramatically increases toward the blue spectral region, exhibiting a strong dispersion. The extinction for most of the frequencies except for the blue edge, displayed in Fig. 2 (b), is not high.

Now consider the evolution of the field intensity along a SPP ray. For certainty, let SPPs propagate along the corresponding rays from the thick edge of the wedge toward the nanofocus as shown in Fig. 1 (b). In the process of such propagation, there will be concentration of the SPP energy in all three directions (3d nanofocusing). This phenomenon differs dramatically from what occurs in conventional photonic ray optics.

To describe this nanofocusing, it is convenient to introduce an orthogonal system of ray coordinates whose unit vectors are $\boldsymbol{\tau}$ (along the ray), $\boldsymbol{\eta}=\left(-\tau_{y}, \tau_{x}\right)$ (at the sur- 
face normal to the ray), and $\mathbf{e}_{z}$ (normal to the surface). The concentration along the ray (in the $\boldsymbol{\tau}$ direction) occurs because the group velocity $v_{g}=\left[\partial\left(k_{0} n\right) / \partial \omega\right]^{-1}$ of SPP asymptotically tends to zero (for the antisymmetric mode) for $k_{0} d \rightarrow 0$ as $v_{g}=v_{0 g} d$ where $v_{0 g}=$ const 13,14 This contributes a factor $A_{\|}=1 / \sqrt{v_{g}(d)}$ to the amplitude of an SPP wave.

The compression of a SPP wave in the $\mathbf{e}_{z}$ (vertical) direction is given by a factor of $A_{z}=\left(\int_{-\infty}^{\infty} W \mathrm{~d} z\right)^{-1 / 2}$, where $W$ is the energy density of the mode. Substituting a standard expression 20 for $W$, one obtains explicitly

$$
\begin{gathered}
A_{z}=\left(\frac{1}{8 \pi} \exp \left(\operatorname{Re} \kappa_{d} d\right)\{\right. \\
\frac{\sinh \left(\operatorname{Re} \kappa_{m} d\right)}{\operatorname{Re} \kappa_{m}\left|\sinh \left(\kappa_{m} d / 2\right)\right|^{2}}\left[1+\frac{d\left(\omega \operatorname{Re} \varepsilon_{m}\right)}{d \omega} \frac{|n|^{2}+\left|\kappa_{m}\right|^{2}}{\left|\varepsilon_{m}\right|^{2}}\right]- \\
\frac{\sin \left(\operatorname{Im} \kappa_{m} d\right)}{\operatorname{Im} \kappa_{m}\left|\sinh \left(\kappa_{m} d / 2\right)\right|^{2}}\left[1+\frac{d\left(\omega \operatorname{Re} \varepsilon_{m}\right)}{d \omega} \frac{|n|^{2}-\left|\kappa_{m}\right|^{2}}{\left|\varepsilon_{m}\right|^{2}}\right]+ \\
\left.\left.\frac{2}{\operatorname{Re} \kappa_{d}}\left[1+\frac{|n|^{2}+\left|\kappa_{d}\right|^{2}}{\varepsilon_{d}}\right]\right\}\right)^{-1 / 2},
\end{gathered}
$$

where $\kappa_{m}=k_{0} \sqrt{n-\varepsilon_{m}}$ and $\kappa_{d}=k_{0} \sqrt{n-\varepsilon_{d}}$.

To obtain the compression factor $A_{\perp}$ for the $\boldsymbol{\eta}$ direction), we consider conservation of energy along the beam of rays corresponding to slightly different values of $n_{x}$. Dividing this constant energy flux by the thickness of this beam in the $\boldsymbol{\eta}$ direction, we arrive at

$A_{\perp}=\left\{\left(1-\frac{n_{x}^{2}}{n^{2}}\right)^{1 / 2} \int_{y_{0}}^{y} \frac{1}{n\left(y^{\prime}\right)}\left[1-\frac{n_{x}^{2}}{n\left(y^{\prime}\right)^{2}}\right]^{-3 / 2} \mathrm{~d} y^{\prime}\right\}^{-1 / 2}$

The ray amplitude thus contains the total factor which describes the 3d adiabatic compression: $A=A_{\|} A_{\perp} A_{z}$.

Now consider the problem of coherent control. The goal is to excite a spatiotemporal waveform at the thick edge of the wedge in such a way that the propagating SPP rays converge at an arbitrary nanofocus at the sharp edge where an ultrashort pulse is formed. To solve this problem, we use the idea of back-propagation or timereversal. 21 We generate rays at the nanofocus as an ultrashort pulse containing just several oscillations of the light field. Propagating these rays, we find amplitudes and phases of the fields at the thick edge at each frequency as given by the eikonal $\Phi(\boldsymbol{\rho})$. Then we complex conjugate the amplitudes of frequency components, which corresponds to the time reversal. We also multiply these amplitudes by $\exp (2 \operatorname{Im} \Phi)$ which pre-compensates for the losses. This provides the required phase and amplitude modulation at the thick edge of the wedge.

We show an example of such calculations in Fig. 3. Panel (a) displays the trajectories of SPPs calculated according to Eq. (4). The trajectories for different frequencies are displayed by colors corresponding to their visual perception. There is a very significant spectral dispersion: trajectories with higher frequencies are much more
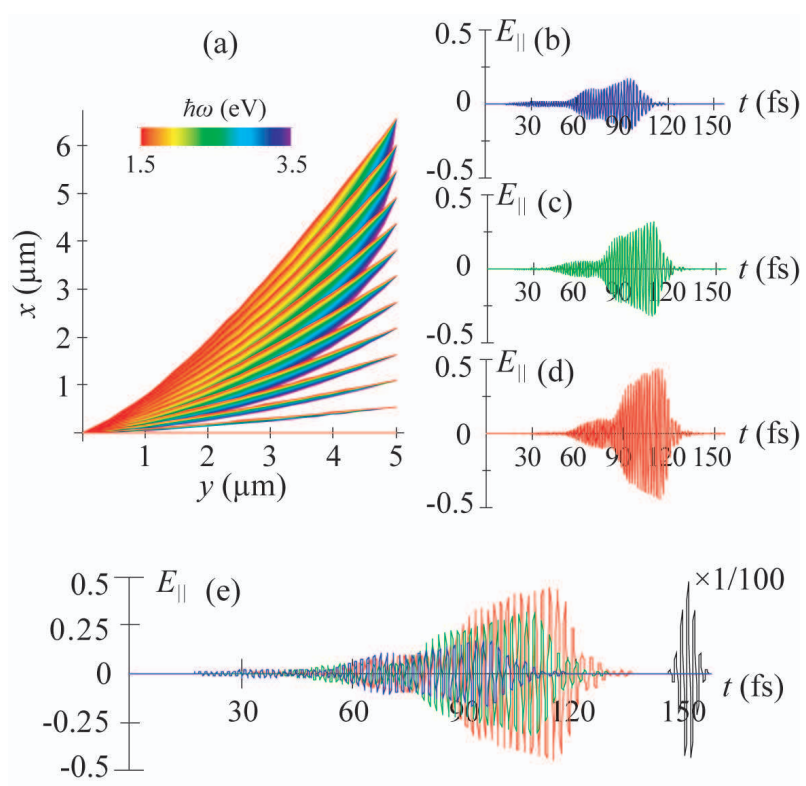

FIG. 3: (a) Trajectories (rays) of SPP packets propagating from the thick edge to the nanofocus displayed in the $x y$ plane of the wedge. The frequencies of the individual rays in a packet are indicated by color as coded by the bar at the top. (b)-(d) Spatiotemporal modulation of the excitation pulses at the thick edge of the wedge required for nanofocusing. The temporal dependencies (waveforms) of the electric field for the phase-modulated pulses for three points at the thick edge boundary: two extreme points and one at the center, as indicated, aligned with the corresponding $x$ points at panel (a). (e) The three excitation pulses of panels (b)-(d) (as shown by their colors), superimposed to elucidate the phase shifts, delays, and shape changes between these pulses. The resulting ultrashort pulse at the nanofocus is shown by the black line. The scale of the electric fields is arbitrary but consistent throughout the figure.

curved. The spatial-frequency modulation that we have found succeeds in bringing all these rays (with different frequencies and emitted at different $x$ points) to the same nanofocus at the sharp edge.

The required waveforms at different $x$ points of the thick edge of the wedge are shown in Fig. [3 (b)-(d) where the corresponding longitudinal electric fields are shown. The waves emitted at large $x$, i.e., at points more distant from the nanofocus, should be emitted significantly earlier to pre-compensate for the longer propagation times. They should also have different amplitudes due to the differences in $A$. Finally, there is clearly a negative chirp (gradual decrease of frequency with time). This is due to the fact that the higher frequency components propagate more slowly and therefore must be emitted earlier to form a coherent ultrashort pulse at the nanofocus.

In Fig. 3 (e) we display together all three of the representative waveforms at the thick edge to demonstrate their relative amplitudes and positions in time. The pulse at the extreme point in $x$ (shown by blue) has the longest way to propagate and therefore is the most advanced in 
time. The pulse in the middle point (shown by green) is intermediate, and the pulse at the center $(x=0$, shown by red) is last. One can notice also a counterintuitive feature: the waves propagating over longer trajectories are smaller in amplitude though one may expect the opposite to compensate for the larger losses. The explanation of this fact is that the losses are actually insignificant for the frequencies present in these waveforms. What determines the relative magnitudes of these waveforms is the coefficient $A_{\perp}$ of the transverse concentration, which is much higher for the peripheral trajectories than for the central ones.

Figure 3 (e) also shows the resulting ultrashort pulse in the nanofocus. This is a transform-limited, Gaussian pulse. The propagation along the rays completely compensates the initial phase and amplitude modulation, exactly as intended. As a result, the corresponding electric field of the waveform is increased by a factor of 100. Taking the other component of the electric field and the magnetic field into account, the corresponding increase of the energy density is by a factor $\sim 10^{4}$ with respect to that of the SPPs at the thick edge.

Consider the efficiency of the energy transfer to the nanoscale. This is primarily determined by the cross section $\sigma_{S P P}$ for scattering of photons into SPPs. For instance, for a metal sphere of radius $R$ at the surface of the wedge, one can obtain an estimate $\sigma_{S P P} \sim R^{6} /\left(d_{m}^{3} \lambda\right)$, where $\lambda$ is the reduced photon wavelength. Setting $R \sim d_{m}$, we estimate $\sigma_{S P P} \sim 3 \mathrm{~nm}^{2}$. Assuming optical focusing into a spot of $\sim 300 \mathrm{~nm}$ radius, this yields the energy efficiency of conversion to the nanoscale of $\sim 10^{-3}$. Taking into account the adiabatic concentration of energy by a factor of $10^{4}$, the optical field intensity at the nanofocus is enhanced by one order of magnitude with respect to that of the incoming optical wave.

The criterion of applicability of the WKB approxima- tion is $\partial k^{-1} / \partial y \ll 1$. Substituting $k=k_{0} n$ and Eq. (5), we obtain a condition $d_{m} /\left(n_{a} L\right)<<1$. This condition is satisfied everywhere including the nanofocus since $n_{a} \sim 1$ and $d_{m} \ll L$ for adiabatic grading. The minimum possible size of the wavepacket at the nanofocus in the direction of propagation, $\Delta x$, is limited by the local SPP wavelength: $\Delta x \sim 2 \pi / k \approx 2 \pi d_{f} / n_{a}$, where $d_{f}$ is the wedge thickness at the nanofocus. The minimum transverse size $a$ (waist) of the SPP beam at the nanofocus can be calculated as the radius of the first Fresnel zone: $a=\pi / k_{x} \geq \pi /\left(k_{0} n_{x}\right)$. Because $n_{x}$ is constant along a trajectory, one can substitute its value at the thick edge (the launch site), where from Eq. (5) we obtain $n_{x} \approx n=n_{a} / d_{m}$. This results in $a \approx \pi d_{m} / n_{a}$; thus $a$ is on order of the maximum thickness of the wedge, which is assumed also to be on the nanoscale.

To briefly conclude, we have proposed and theoretically investigated an approach to full coherent control of spatiotemporal energy localization on the nanoscale. From the thick edge of a plasmonic metal nanowedge, SPPs are launched, whose phases and amplitudes are independently modulated for each constituent frequency of the spectrum and at each spatial point of the excitation. This pre-modulates the departing SPP wave packets in such a way that they reach the required point at the sharp edge of the nanowedge in phase, with equal amplitudes forming a nanofocus where an ultrashort pulse with required temporal shape is generated. This system constitutes a "nanoplasmonic portal" connecting the incident light field, whose features are shaped on the microscale, with the required point or features at the nanoscale.

This work was supported by grants from the Chemical Sciences, Biosciences and Geosciences Division of the Office of Basic Energy Sciences, Office of Science, U.S. Department of Energy, a grant CHE-0507147 from NSF, and a grant from the US-Israel BSF.
1 M. Shapiro and P. Brumer, Physics Reports 425, 195 (2006).

2 H. Rabitz, R. de Vivie-Riedle, M. Motzkus, and K. Kompa, Science 288, 824 (2000).

3 A. Apolonski, P. Dombi, G. G. Paulus, M. Kakehata, R. Holzwarth, T. Udem, C. Lemell, K. Torizuka, J. Burgdoerfer, T. W. Hansch, et al., Phys. Rev. Lett. 92, 073902 (2004).

4 D. Zeidler, A. Staudte, A. B. Bardon, D. M. Villeneuve, R. Dorner, and P. B. Corkum, Phys. Rev. Lett. 95, 203003 (2005).

5 M. I. Stockman, S. V. Faleev, and D. J. Bergman, Phys. Rev. Lett. 88, 067402 (2002).

6 M. I. Stockman, D. J. Bergman, and T. Kobayashi, Phys. Rev. B 69, 054202 (2004).

7 M. I. Stockman and P. Hewageegana, Nano Lett. 5, 2325 (2005)

8 A. Kubo, K. Onda, H. Petek, Z. Sun, Y. S. Jung, and H. K. Kim, Nano Lett. 5, 1123 (2005).

9 M. Sukharev and T. Seideman, Nano Lett. 6, 715 (2006).
10 M. Aeschlimann, M. Bauer, D. Bayer, T. Brixner, F. J. G. d. Abajo, W. Pfeiffer, M. Rohmer, C. Spindler, and F. Steeb, Nature 446, 301 (2007).

11 M. M. Wefers and K. A. Nelson, Opt. Lett. 18, 2032 (1993).

12 T. Feurer, J. C. Vaughan, and K. A. Nelson, Science 299, 374 (2003).

13 M. I. Stockman, Phys. Rev. Lett. 93, 137404 (2004).

14 M. I. Stockman, in Plasmonics: Metallic Nanostructures and Their Optical Properties II, edited by N. J. Halas and T. R. Huser (SPIE, Denver, Colorado, 2004), vol. 5512, pp. 38-49.

15 A. J. Babajanyan, N. L. Margaryan, and K. V. Nerkararyan, J. Appl. Phys. 87, 3785 (2000).

16 S. A. Maier, S. R. Andrews, L. Martin-Moreno, and F. J. Garcia-Vidal, Phys. Rev. Lett. 97, 176805 (2006).

17 D. K. Gramotnev, J. Appl. Phys. 98, 104302 (2005).

18 E. Verhagen, L. Kuipers, and A. Polman, Nano Lett. (2006).

19 P. B. Johnson and R. W. Christy, Phys. Rev. B 6, 4370 (1972). 
${ }^{20}$ L. D. Landau and E. M. Lifshitz, Electrodynamics of Continuous Media (Pergamon, Oxford and New York, 1984).

315, 1120 (2007).

${ }^{21}$ G. Lerosey, J. de Rosny, A. Tourin, and M. Fink, Science 